This item was submitted to Loughborough's Research Repository by the author.

Items in Figshare are protected by copyright, with all rights reserved, unless otherwise indicated.

\title{
Atomistic modelling of titania grown using PVD methods
}

PLEASE CITE THE PUBLISHED VERSION

http://dx.doi.org/10.1109/PVSC.2012.6318059

PUBLISHER

(c) IEEE

VERSION

AM (Accepted Manuscript)

LICENCE

CC BY-NC-ND 4.0

REPOSITORY RECORD

Blackwell, Sabrina, Roger Smith, Steven D. Kenny, and Michael Walls. 2019. "Atomistic Modelling of Titania Grown Using PVD Methods". figshare. https://hdl.handle.net/2134/11616. 
This item was submitted to Loughborough's Institutional Repository (https://dspace.lboro.ac.uk/) by the author and is made available under the following Creative Commons Licence conditions.

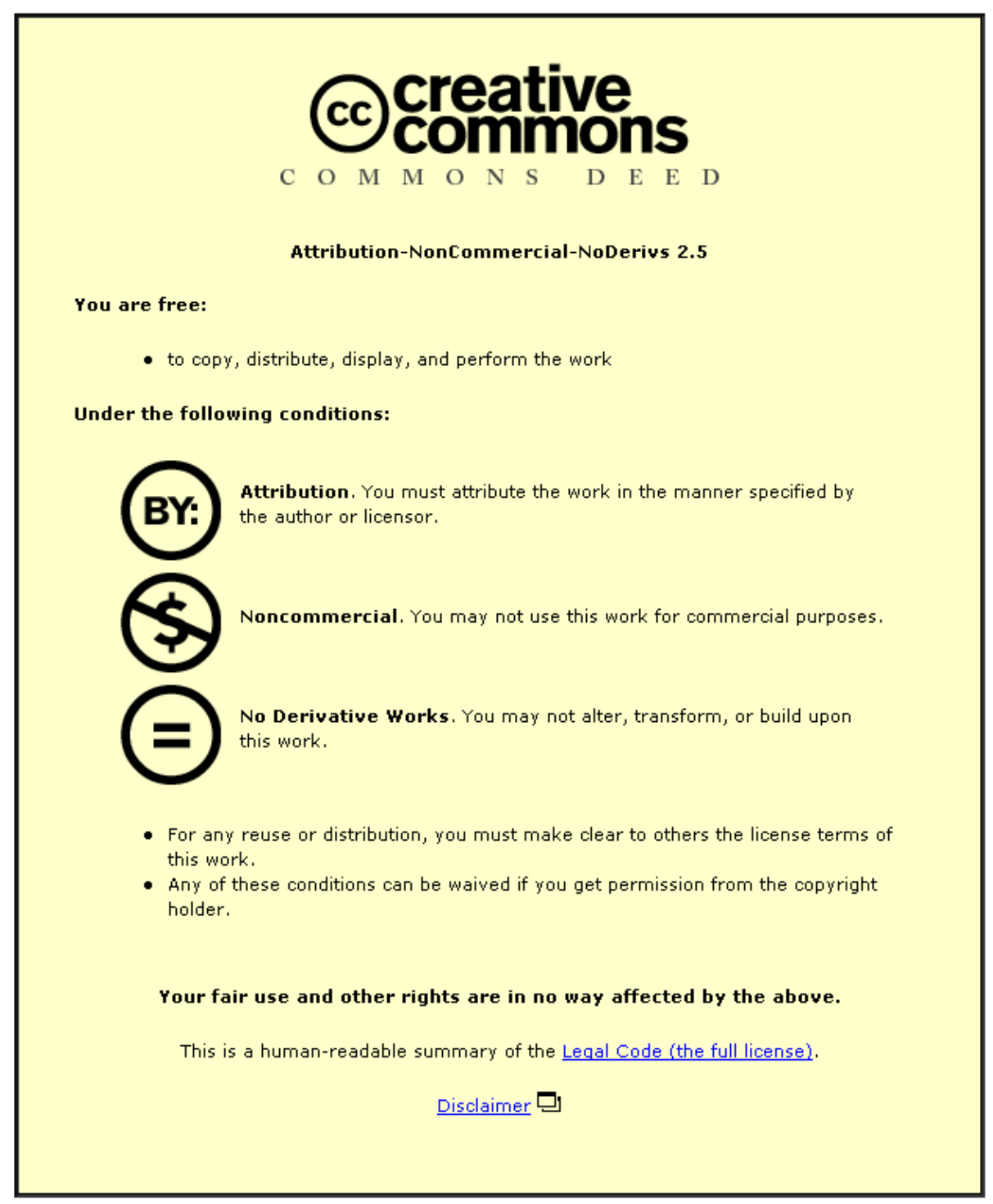

For the full text of this licence, please go to: http://creativecommons.org/licenses/by-nc-nd/2.5/ 


\title{
Atomistic Modelling of Titania Grown using PVD Methods
}

\author{
Sabrina Blackwell ${ }^{1}$, Roger Smith ${ }^{1}$, Steven D. Kenny ${ }^{1}$ and John M. Walls ${ }^{2}$ \\ ${ }^{1}$ Department of Mathematical Sciences and ${ }^{2}$ Department of Electronic and Electrical Engineering, \\ Loughborough University, Loughborough, Leicestershire, LE11 3TU, UK
}

\begin{abstract}
Results are presented for the atomistic modelling of titania growth, specifically the rutile $\{110\}$ surface. Long time scale dynamics techniques are used to model the growth of thin films at realistic growth rates. Between deposition events, the system is evolved through an on-the-fly Kinetic Monte Carlo (otf-KMC) method, finding diffusion pathways and barrier heights without any prior knowledge of transitions. OtfKMC allows thorough investigation of transitions and barriers observed during the film growth, giving a deeper understanding of growth mechanisms. An important rutile growth mechanism observed during all simulations, involves the upward diffusion of $\mathrm{Ti}$ interstitials below an $\mathrm{O}$ rich surface, with a barrier of $0.61 \mathrm{eV}$. The energy required for a single $O$ ad-atom to diffuse on the surface is higher (between $0.65 \mathrm{eV}-0.85 \mathrm{eV}$ ). Methods used also allow examination of the effects of varying the experimental parameters, such as substrate bias, plasma density and stoichiometry of the deposited material. Conclusions drawn from the film growth suggest that the evaporation process produces an incomplete structure with voids, which is interesting for dye cell use. The inclusion of a low energy ion-beam assist does however add enough kinetic energy to the substrate to enable the completion and densification of layers, producing a film with greater crystallinity. The sputtering process produces highly crystalline growth, which is useful for anti-reflection coatings.
\end{abstract}

Index Terms-Titanium dioxide, thin film, evaporation, sputtering, ion assist, modelling, dye sensitized solar cells.

\section{INTRODUCTION}

Titania in its rutile form is an important material with industrial scale applications. Uses range from pigment to sunscreen to photovoltaic devices, and it is the latter which is the focus of this work. The high refractive index and UV radiation absorption abilities of dense, crystalline $\mathrm{TiO}_{2}$ films lends to multilayer optical coating uses [1]. $\mathrm{TiO}_{2}$ in its porous form is required for dye sensitized solar cells [2].

Thin films, specifically metal-oxide films in this case, can be deposited using numerous industrial-scale processes [3]. Evaporation, ion-beam assisted evaporation [4], [5], [6] and reactive magnetron sputtering [7], [8], [9] are typical processes used. The deposition rate of $\mathrm{TiO}_{2}$ in industry is typically anywhere between $1 / 2-2$ monolayers per second; here we use 1/2 monolayer per second. Evaporation deposition is simulated by deposition of $\mathrm{TiO}_{x}$ ad-clusters onto the rutile $\{110\}$ surface, arriving with typical kinetic energy of $<1 \mathrm{eV}$. An ion source, in this case Ar, is used to aid the densification of the film and to introduce extra energy into the substrate in order to increase atomic mixing and surface diffusion. The use of both low and medium energy ion sources was simulated, where Ar arrived at the surface with either $40 \mathrm{eV}$ or $100 \mathrm{eV}$ kinetic energy respectively. The arrival rate of $\mathrm{Ar}$ was matched to that of the $\mathrm{TiO}_{x}$ ad-clusters, equivalent to $1.6 \times 10^{15}$ atoms $/ \mathrm{cm}^{2} / \mathrm{s}$, it is expected that most of the Ar will leave the surface during the simulation. Sputter deposition deposits $\mathrm{TiO}_{2}$ thin films using either RF, DC or pulsed DC power. Targets in the magnetron can be $\mathrm{TiO}_{2}$ (RF), Ti (DC),or $\mathrm{TiO}_{x}$ (pulsed DC). As with evaporation, $\mathrm{TiO}_{x}$ ad-clusters arrive at the surface. The ad-units are sputtered from the target and arrive at the substrate with increased kinetic energy, typically $40 \mathrm{eV}$. Ar present within the magnetron is used as the working gas in order to sputter the material from the target, thus it is know that Ar will also bombard the substrate with a similar kinetic energy $(40 \mathrm{eV})$. This Ar bombardment during deposition is known to affect the density and stoichiometry of the resulting oxide film [10]. Ar impacts on the substrate can also transfer momentum thus enhance surface mobility and diffusion, increasing film quality, leading to a higher refractive index [11].

Atomistic simulation has been used to complement experiment to understand the mechanisms involved. However, despite advances in computational power, using traditional Molecular Dynamics (MD) alone to model systems over long time scales is computationally infeasible. Traditional MD allows simulation for a few milliseconds, whereas the time required to grow one monolayer of $\mathrm{TiO}_{2}$ is $\backsim 2$ seconds. This is because a typical time step in the numerical integration scheme is around $1 \times 10^{-15} \mathrm{~s}$. Thus to simulate the deposition of one monolayer would require $10^{16}$ integration time steps. Each step requires the evaluation of the forces on all the atoms and is computationally expensive, especially for the variable charge model used here. Thus it is not possible to simulate thin film growth over realistic time scales using MD alone. If the deposition rate is artificially enhanced to allow the MD simulations to be carried out, then the system dynamics is biased. Long time scale dynamics combines MD with new techniques, allowing simulation over experimental time scales without biasing the system dynamics. On-the-fly Kinetic Monte Carlo (otf-KMC) [12] is combined with MD allowing simulation of systems over several seconds of real time. Otf-KMC methods also allow detailed investigation of individual transitions, enabling a deeper understanding of the growth mechanisms involved.

\section{Methodology}

Simulations were carried out using a modified variable charge $\mathrm{TiO}_{2}$ interatomic potential [13], [14]. The original 
potential, by Hallil et al. [15] was modified on the basis of ab-initio calculations [13] that were carried out to determine energy migration barriers. It was found that the original potential gave a much higher barrier for subsurface $\mathrm{Ti}$ interstitials to migrate to the surface and this migration mechanism was found to be one that was crucial to the way in which growth could take place. Periodic boundary conditions were employed for the substrate, mimicking the bulk in the lateral direction. The bottom layer of atoms was held fixed and the two layers above connected to a heat bath, maintained at a temperature of $350 \mathrm{~K}$. Typical system sizes were 5 monolayers deep with $\backsim 200$ atoms in each layer, simulations beginning with a perfect $\{110\}$ rutile surface. An ad-unit was deposited onto the surface with a prescribed energy by running MD for up to $10 \mathrm{ps}$, allowing the lattice return to $350 \mathrm{~K}$ and defects to stabilize. The system was then relaxed and diffusion between deposition events was simulated using otf-KMC. At each step either a deposition event or a diffusion event is chosen, according to their relative probabilities. Growth continues in this way until we have grown the number of layers we wish. The key point here is that the growth technique involves MD and otf-KMC working together.

During deposition, a variety of small molecular species arrive at the surface. We assume that these consist of a mixture of $\mathrm{O}$ and $\mathrm{Ti}$ atoms, $\mathrm{O}_{2}$ molecules, $\mathrm{TiO}$ and $\mathrm{TiO}_{2}$ units, resulting in the stoichiometric deposition of $\mathrm{TiO}_{2}$. These are assumed to impact normally on the surface with an energy of $1 \mathrm{eV}$ for the model of e-beam deposition and with energies that vary up to $40 \mathrm{eV}$ for modelling magnetron sputtering. A very useful part of the simulation is that the species mix and energy can be varied in the simulation to determine the most appropriate stoichiometry and energy for crystalline growth. In addition to the deposition species $\mathrm{Ar}$ atoms can also be introduced into the model. In magnetron sputtering these will have a similar energy to the deposition species but in ion beam assist the energy can be much higher. In our model we investigate simultaneous Ar bombardment also at normal incidence up to energies of $100 \mathrm{eV}$.

Otf-KMC specifically calculates all available transitions onthe-fly, enabling us to simulate much more defective and complicated systems. First, defects are identified along with all neighbouring atoms. This defines a defect region in which transitions from one local minimum to another are sought. Next, transition (saddle) points surrounding a metastable state (local minimum) are approximately located using a saddle point searching method [14]. Finally the climbing image Nudged Elastic Band method (NEB) [16] determines the barrier heights, more exactly. Typically $>200$ searches are carried out per step. The transition frequency $\nu$, of every unique transition is determined using the Arrhenius equation

$$
\nu=\nu_{0} \exp (-E / k T)
$$

with an assumed constant prefactor $\nu_{0}$, usually taken as $10^{12}$, where $E$ is the barrier height, $k$ is Boltzmanns constant and $T$ is temperature. Transition searches together with a deposition event are carried out in parallel, on typically between 24-48 cpu cores, where the deposition event runs MD on a single core and searches are employed on multiple cores. Either a transition or deposition event is chosen from a roulette algorithm, which is used to evolve the system in time. Further details of the algorithm can be found elsewhere [12].

\section{RESULTS}

\section{A. Typical Growth Mechanisms}

Titania growth quality is dependant on the deposition process used, however, there are growth mechanisms observed during all processes that allow surface diffusion and more complete structures to form. A key feature of the deposition process is that $\mathrm{O}$ atoms and $\mathrm{O}_{2}$ molecules nearly always attach to the surface whereas isolated $\mathrm{Ti}$ atoms almost invariably implant below the surface. Figure 1 illustrates a simple example of the rutile growth mechanism, a mechanism aiding the growth of titania via the migration of Ti interstitials up to the surface when surface is O rich [17], [18]. This is a key mechanism in enabling completion of layers and the annealing of $\mathrm{Ti}$ interstitials [13].

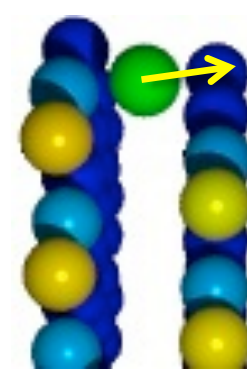

21.0
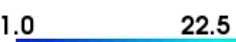

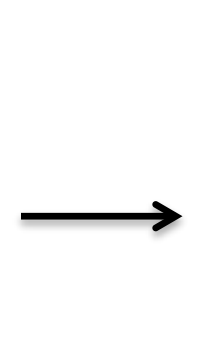

Height in $Y(A)$ 24.0
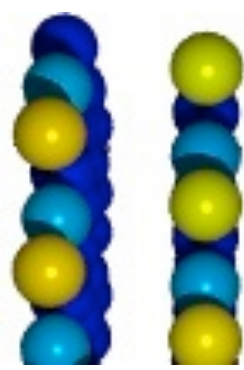

25.5
Fig. 1. Example of a rutile growth mechanism. Only the Ti atoms are shown; a $\mathrm{Ti}$ in an interstitial site diffuses upwards to fill a Ti vacancy in the surface with an energy barrier of $0.61 \mathrm{eV}$. There is $\mathrm{O}$ present on the surface above the Ti vacancy, this reduces the transition barrier and helps the Ti to diffuse upwards. Atoms are coloured by height using the key.

More intuitive transitions also take place to enable growth to occur; single $\mathrm{O}$ ad-atoms, for example, diffuse on the surface with energy barriers of $0.65 \mathrm{eV}-0.85 \mathrm{eV}$. The lower range of these barriers result in the diffusion of $\mathrm{O}$ ad-atoms along the trenches, above the 5-fold co-ordinate $\mathrm{Ti}$ row, as illustrated in figure 2. If the $\mathrm{O}$ ad-atoms adhere above a $\mathrm{Ti}$ interstitial this lowers the energy barrier for the Ti interstitial to diffuse to the surface, this enables the formation of $\mathrm{TiO}_{2}$ units on the surface. With slightly higher energy barriers, the $\mathrm{O}$ ad-atom can fill any vacancies in the $\mathrm{O}$ ad-rows, aiding the completion of layers as shown in figure 3 . 


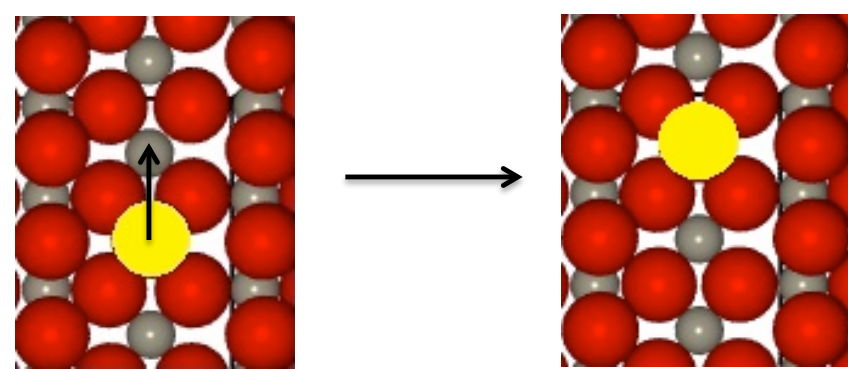

Fig. 2. An $\mathrm{O}$ ad-atom sitting above 5-fold $\mathrm{Ti}$ in the trench diffuses up and down the trench with energy barriers ranging typically between $0.65 \mathrm{eV}$ and $0.75 \mathrm{eV}$. Ad-atoms may diffuse towards a Ti interstitial, thus lowering the energy required for the interstitial to diffuse upwards and join the $\mathrm{O}$ ad-atom as a ad-cluster on the surface. Larger, red spheres represent $\mathrm{O}$ and the smaller, grey spheres represent $\mathrm{Ti}$.
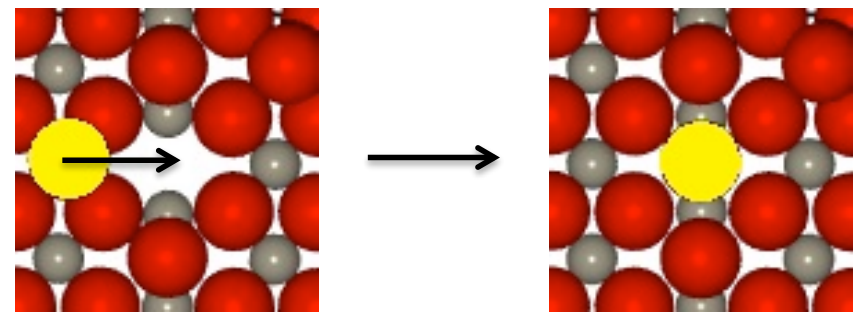

Fig. 3. An O ad-atom sitting above 5-fold co-ordinate $\mathrm{Ti}$ in the trench moves across to fill a vacancy in the $\mathrm{O}$ ad-row. This takes place with an energy barrier of $0.81 \mathrm{eV}$. Transitions similar to this enable layers to become complete.

The mechanisms shown in figures 1, 3 are the most important in terms of producing good crystalline growth but there are also many other concerted mechanisms that take place. Figure 4 illustrates the concerted movement of an ad-unit on the surface. The $\mathrm{O}$ ad-row has a $\mathrm{TiO}_{2}$ ad-cluster joined to it, with a $\mathrm{O}$ ad-atom on top. The otf-KMC methods allow investigation of these concerted, multi atom transitions. The $\mathrm{O}$ atoms rotate around a point to effectively slide the unit down the trench. Transitions such as this allow layer by layer growth to occur as this unit would perhaps enable the drawing out of the Ti interstitials below.

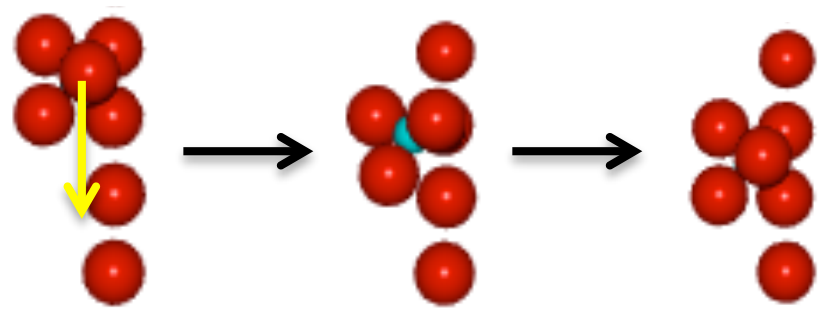

Fig. 4. $\mathrm{A} \mathrm{TiO}_{2}$ unit with a $\mathrm{O}$ sitting on top diffuses down the trench above the 5 -fold coordinated Ti row with a barrier of $0.83 \mathrm{eV}$. This image shows only the involved atoms, along with the neighbouring $\mathrm{O}$ ad-row, to avoid confusion.

However, the barriers for these mechanisms are relatively high compared to the growth of metal films [19]. This implies that they require some added energy into the system to activate. This is discussed in the subsequent sections of the paper where we consider first evaporation growth without any added energy and then two experimental deposition methods that do add energy to the system. The various resulting growth structures are compared.

\section{B. Evaporation Deposition and Ion-Beam Assist}

Four monolayers of atoms have been deposited onto the substrate via the evaporation process, simulating up to 6 seconds of experimental time. Ad-units arrive at the substrate with kinetic energy of $<1 \mathrm{eV}$. During ion-beam assist, $\mathrm{Ar}$ arrives at the same rate and at either low or high energy (40 $\mathrm{eV}$ or $100 \mathrm{eV}$ ). Growth from evaporation deposition alone, without ion-beam assist, produces highly incomplete layers, with both oxygen and titanium atoms missing. Low kinetic energy of the arriving ad-clusters does not transfer sufficient energy to the substrate for much surface diffusion to occur, thus leaving the layers incomplete and void filled.

Both low and high energy ion-beam assist on the evaporation growth have been simulated. It is clear that the addition of low energy ion-beam assist (Ar arriving with $40 \mathrm{eV}$ kinetic energy) produces layers which are more crystalline, as illustrated in figure 5 The Ar depositions introduce more energy into the system thus allowing more mixing, diffusion and growth mechanisms to take place, producing a more crystalline structure to form over time. The higher energy ion-beam assist (Ar arriving with $100 \mathrm{eV}$ kinetic energy), however, introduces too much energy to the system, causing damage to the surface and producing voids, thus disrupting the crystallinity whilst appearing to densify the film. Implantation of Ar into the substrate is observed during the ion-beam assist, with deep implantation from the higher energy Ar bombardments, up to 4 layers deep into the substrate. From the experimental results it was expected that the ion-beam assist would improve film density [8], however, from our calculations an increased density effect cannot be definitely concluded, as growth has not yet reached a steady state.

Ar within the lattice preferentially sits in one of two locations; in an interstitial location between layers or as a substitutional atom in a $\mathrm{Ti}$ site after knocking the $\mathrm{Ti}$ into a deeper interstitial site within the lattice. The higher energy $\mathrm{Ar}$ assist creates increased $\mathrm{Ti}$ interstitials deeper within the lattice than the less disruptive $40 \mathrm{eV}$ ion assist. The rutile growth mechanism allows some $\mathrm{Ti}$ interstitials to anneal, however, $\mathrm{Ti}$ interstitials very deep within the lattice require more time to diffuse upwards and thus often remain stuck in the lattice over the time to grow one or two monolayers.

\section{Sputter Deposition}

Four monolayers of atoms have been deposited via magnetron sputtering, where ad-units arrive at the substrate with $40 \mathrm{eV}$. Biasing the substrate is possible in order to increase the 


\section{Evaporation}

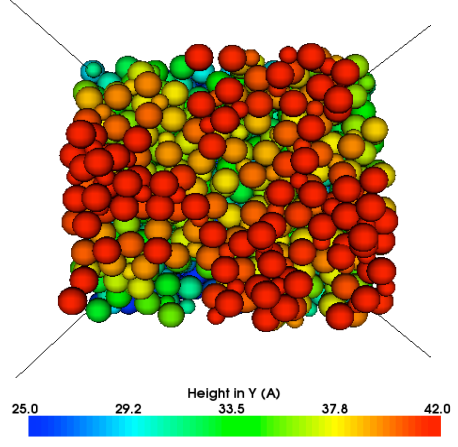

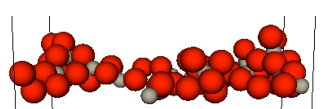

\section{Low energy ion-beam assist}
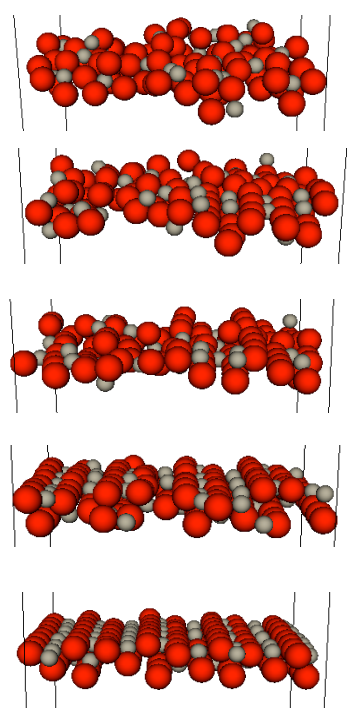
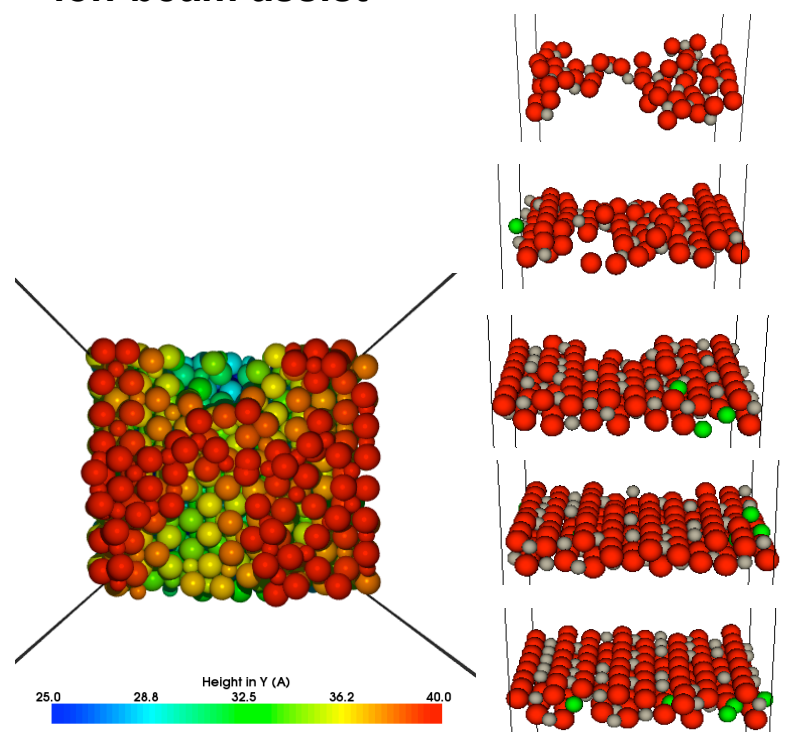

Fig. 5. Comparison of growth of $\mathrm{TiO}_{2}$ from evaporation and low energy ion-beam assisted evaporation, where Ar arrived at the surface with equal probability as a $\mathrm{TiO}_{x}$ cluster and with $40 \mathrm{eV}$. Both surfaces are shown, with a breakdown of the newly grown layers. Evaporation produces a void filled, incomplete film whereas the ion-beam assist adds enough energy to the system for more complete layers to form thanks to increased diffusion. Atoms in the surface image are coloured by height using the key. Larger, red spheres represent O, the smaller, grey spheres represent Ti and small, green spheres represent Ar left in the film.

deposition energy as high as $100 \mathrm{eV}$. However, depositing at such high energy actually damages the substrate too much, resulting in a highly incomplete, porous structure with $\mathrm{Ti}$ interstitials as deep as the 4th layer, too deep for the rutile growth mechanism to be able to draw them out. Optical properties of the film would significantly alter in this case, therefore only the $40 \mathrm{eV}$ sputter deposition case is discussed. Simulation of the sputtering process of $\mathrm{TiO}_{2}$, firstly ignoring the effect of $\mathrm{Ar}$ in the plasma, produces a highly crystalline and complete film, shown in figure 6 . The $40 \mathrm{eV}$ depositions transfer enough kinetic energy to the substrate to allow surface diffusion to occur and $\mathrm{O}$ atoms present on the surface enable the rutile growth mechanism to take place promoting increased crystallinity. It is clear that in the magnetron sputtering case, Ar does not cause any dramatic changes in the quality of growth produced as results are very similar, the only difference being that $\mathrm{Ar}$ bombardments disrupt the original substrate more thus enhancing atomic mixing. Experimental results report that Ar impacts improve film density, however, as our simulations have not reached a steady state no firm conclusions can be drawn. Ar bombardment produced a higher proportion of Ti interstitials in the substrate, which would aid the rutile growth mechanism in forming more crystalline rutile, however, a significant difference in growth quality is not evident.

\section{CONCLUSIONS}

Otf-KMC partnered with traditional MD allows simulation of thin film growth over realistic, experimental time scales. In this work, simulations have modelled up to 6 seconds of film growth; using traditional MD this would require millions

\section{Sputtering}
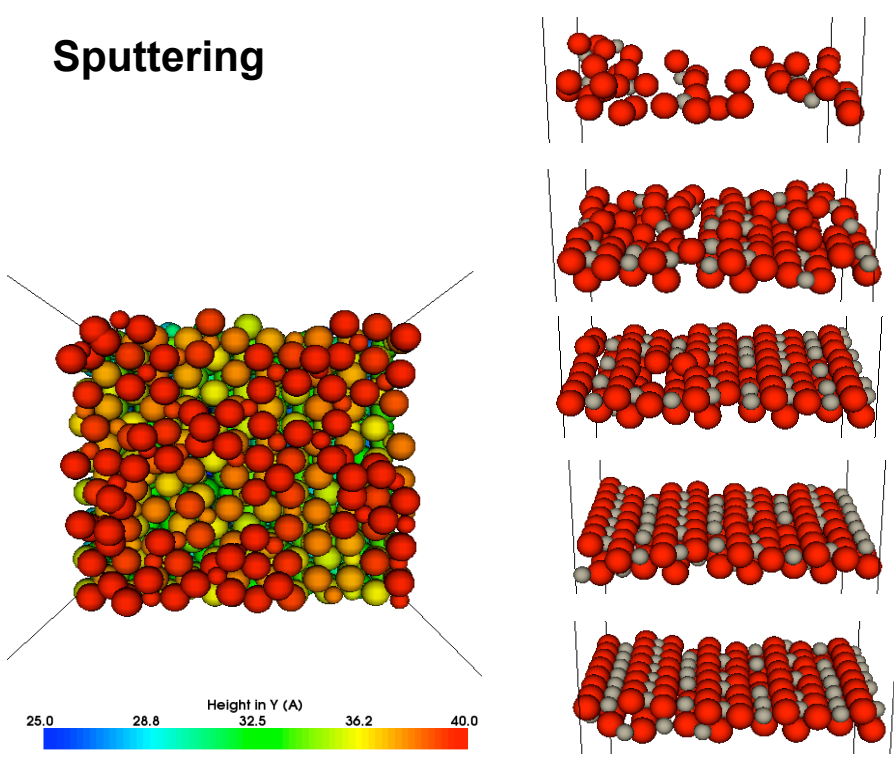

Fig. 6. Growth of $\mathrm{TiO}_{2}$ from magnetron sputtering, where atoms arrive at the substrate with $40 \mathrm{eV}$. The surface in shown with a breakdown of new layers, which are highly crystalline and complete. The higher energy depositions have enabled surface diffusion and mixing to produce this crystalline film. Atoms in the surface image are coloured by height using the key. Larger, red spheres represent $\mathrm{O}$, and smaller, grey spheres represent $\mathrm{Ti}$.

of computing hours.

The otf-KMC methods allow thorough investigation of transitions observed and growth mechanisms involved. The 
rutile growth mechanism, shown in figure 1, is one of the most important observed; $\mathrm{Ti}$ interstitials below the surface are drawn upwards by the presence of an $\mathrm{O}$ rich surface. This key mechanism encourages the annealing of $\mathrm{Ti}$ interstitials and the completion of layers above. With a barrier of 0.61 $\mathrm{eV}$, this mechanism is observed during all simulations; but of course the deposition processes transferring the most kinetic energy to the substrate will encourage a higher diffusion rate, however $\mathrm{Ti}$ interstitials must be only in the first layer for the mechanism to work. Diffusion of single $\mathrm{O}$ ad-atoms was also investigated and found to occur requiring slightly higher energy $(0.65 \mathrm{eV}-0.85 \mathrm{eV}) . \mathrm{O}$ ad-atoms were found to diffuse up and down the trench, above the 5-fold Ti rows, enabling units to form and hence completion of layers. Vacancies in the $\mathrm{O}$ ad-rows noticeably are filled in by the same $\mathrm{O}$ ad-atoms which may be diffusing up and down the trenches. Understanding specific mechanisms occurring during growth allows a deeper understanding of the growth itself.

Evaporation deposition was modelled by depositing adclusters with $<1 \mathrm{eV}$ kinetic energy onto the rutile $\{110\}$ surface. Incomplete layers were grown with voids throughout, as illustrated in figure 5. With such low kinetic energy involved in the depositions, little surface diffusion occurs, hence leaving layers incomplete and porous. Inclusion of the low energy $(40 \mathrm{eV})$ ion-beam assist transfers increased kinetic energy to the substrate, encouraging increased surface diffusion and thus a more crystalline and complete structure. Ion-beam assist, both low and high energy, produced denser films, although as the system has not reached a steady state we cannot be sure that this is a real effect. For a better insight growth would need to continue for several more layers.

Sputter deposition was modelled by depositing ad-clusters with $40 \mathrm{eV}$ kinetic energy onto the surface; this was found to be the optimal deposition energy to produce dense and crystalline thin films [17], [20]. Resulting growth was crystalline and almost complete. The $\mathrm{Ar}$ within the working gas in the magnetron was included in simulations by bombarding with $\mathrm{Ar}$ at the same rate as the $\mathrm{TiO}_{x}$ and with the same deposition energy of $40 \mathrm{eV}$. Ar bombardments lead to an increase in mixing with the original substrate via damage and repair; an increase in $\mathrm{Ti}$ interstitials was also observed, however none of these changes improved the quality of the film significantly. From experimental reports it was thought that Ar impacts would increase film density, however the systems have not yet reached steady states so no clear conclusions can be drawn on density improvement.

\section{ACKNOWLEDGMENT}

The authors would like to thank EPSRC and TSB for financial support and Loughborough University for the High Performance Computing time. One of the authors (S. Blackwell) thanks Loughborough University for a postgraduate studentship. This work was funded by EPSRC grant no. EP/C524322/1.

\section{REFERENCES}

[1] Z. Zhang, W. A. Anderson, and M. Moo-Young, "Rigorous modeling of uv absorption by $\mathrm{TiO}_{2}$ films in a photocatalytic reactor," American Institute of Chemical Engineers Journal, vol. 46, pp. 1461-1470, (2000).

[2] H. Wang, C. Su, H. Chen, Y. Liu, Y. Hsu, N. Hsu, and W. Li, "Preparation of nanoporous $\mathrm{TiO}_{2}$ electrodes for dye-sensitized solar cells," Journal of Nanomaterials, vol. 2011, p. 7, (2011).

[3] D. M. Mattox, Handbook of Physical Vapor Deposition (PVD) Processing, 2nd ed. Elsevier Science Ltd, 2010.

[4] D. E. Wolfe and J. Singh, "Microstructural evolution of titanium nitride TiN coatings produced by reactive ion beam-assisted, electron beam physical vapor deposition RIBA, EB-PVD," Journal of Materials Science, vol. 34, pp. 2997-3006, (1999).

[5] K. Bange, C. R. Ottermann, O. Anderson, U. Jeschkowski, M. Laube, and $\mathrm{R}$. Feile, "Investigations of $\mathrm{TiO}_{2}$ films deposited by different techniques," Thin Solid Films, vol. 197, pp. 279-285, (1991).

[6] F. Zhang, S. Jin, Y. Mao, Z. Zheng, Y. Chen, and X. Liu, "Surface characterization of titanium oxide films synthesized by ion beam enhanced deposition," Thin Solid Films, vol. 310, pp. 29-33, (1997).

[7] S. H. Kim, J. H. Lee, C. K. Hwangbo, and S. M. Lee, "DC reactive magnetron sputtering with Ar ion-beam assistance for titanium oxide films," Surface and Coatings Technology, vol. 158-159, pp. 457-464, (2002).

[8] P. Zeman and S. Takabayashi, "Nano-scaled photocatalytic $\mathrm{TiO}_{2}$ thin films prepared by magnetron sputtering," Thin Solid Films, vol. 433, pp. 57-62, (2003).

[9] J. Sicha, J. Musil, M. Meissner, and R. Cerstvy, "Nanostructure of photocatalytic $\mathrm{TiO}_{2}$ films sputtered at temperatures below 200C," Applied Surface Science, vol. 254, pp. 3793-3800, (2008).

[10] K. Eufinger, E. N. Janssen, H. Poelman, D. Poelman, R. De Gryse, and G. B. Marin, "The effect of argon pressure on the structural and photocatalytic characteristics of tio 2 thin films deposited by d.c. magnetron sputtering," Thin Solid Films, vol. 515, pp. 425-429, 2006.

[11] S. H. Kim and C. K. Hwangbo, "Influence of ar ion-beam assistance and annealing temperatures on properties of tio 2 thin films deposited by reactive dc magnetron sputtering," Thin Solid Films, vol. 475, pp. 155-159, 2005.

[12] C. Scott, S. Blackwell, L. J. Vernon, S. D. Kenny, J. M. Walls, and R. Smith, "Atomistic surface erosion and thin film growth modelled over realistic time scales," Journal of Chemical Physics, vol. 135, p. 174706, 2011

[13] L. J. Vernon, "Modelling the growth of tio 2 ," Ph.D. dissertation, Loughborough University, 2010.

[14] E. J. Sanville, L. J. Vernon, S. D. Kenny, R. Smith, Y. Moghaddam, C. Browne, and P. Mulheran, "Surface and interstitial transition barriers in rutile (110) surface growth," Physical Review B, vol. 80, p. 235308 , 2009.

[15] A. Hallil, R. Tetot, F. Berthier, I. Braems, and J. Creuze, "Use of a variable-charge interatomic potential for atomistic simulations of bulk, oxygen vacancies, and surfaces of rutile tio 2 ," Physical Review B, vol. 73 , p. 165406,2006

[16] G. Henkelman, D. Sheppard, and R. Terrell, "Optimization methods for finding minimum energy paths," Journal of Chemical Physics, vol. 128 pp. 134 106(1-10), 2008.

[17] L. J. Vernon, S. D. Kenny, R. Smith, and E. J. Sanville, "Growth mechanisms for tio 2 at its rutile (110) surface," Physical Review B, vol. 83, p. 075412, 2011.

[18] S. Wendt, P. T. Sprunger, E. Lira, G. K. H. Madsen, Z. Li, J. Hansen, J. Matthiesen, A. Blekinge-Rasmussen, E. Lægsgaard, B. Hammer, and F. Besenbacher, "The role of interstitial sites in the ti3d defect state in the band gap of titania," Science, vol. 320, p. 1755, 2008.

[19] S. Blackwell, R. Smith, S. D. Kenny, and J. M. Walls, "Modelling evaporation, ion-beam assist and magnetron sputtering of thin metal films over realistic timescales," 2012, manuscript submitted for publication.

[20] L. J. Vernon, S. D. Kenny, and R. Smith, "Growth of $\mathrm{TiO}_{2}$ surfaces following low energy ( $\leq 40 \mathrm{ev})$ atom and small cluster bombardment," Nuclear Instruments and Methods in Physics Research B, vol. 268, pp. 2942-2946, (2010). 